\title{
Analysis of Electric Bionic Stimulation(EBS)'s Effect on Insomnia of Quinquagenarian
}

\author{
He Zhilei $^{1, a, *}$, Yang Xiuxian ${ }^{2, b}$, Zhang Yujia ${ }^{1, c}$, Qi Jinling ${ }^{1, d}$, Han Guochao,e \\ ${ }^{1}$ Qiqihar Medical University, Qiqihar Pukui Street,Qiqihar ,China \\ ${ }^{2}$ Harbin Medical University, Haerbin Baojian Street, Haerbin, China \\ a69724849@qq.com, ${ }^{\text {b } 396171469 @ q q . c o m, ~}{ }^{\mathrm{c}}$ 87605009@qq.com, ${ }^{\mathrm{d}}$ \\ 113348606@qq.com e253439565@qq.com \\ *corresponding author
}

Keywords: Electric bionic stimulation, Statistical method, insomnia

\begin{abstract}
This article aims to study EBS's effect on clinical treatment of insomnia. Choose 80 patients who accord with insomnia diagnostic criteria of ICT-10 and divide them into experimental group and matched group at random. Experimental group take a routine way of medicine and matched group take EBS therapy besides medicine.Before treatment,we use PSQI to take baseline evaluation,therapeutic evaluation and evaluate the index of security. Two groups of patients' scores of PSQI have a obvious descend after treatment and have a statistics meaning compared with those taken before treatment $(\mathrm{P}<0.05)$. There are five indexes of PSQI in experimental group lower than that in matched group after treatment,which has the statistic meaning if comparing the two groups $(\mathrm{P}<0.05)$ and progress of sleep attains an improvement. EBS does have an effect on treatment of quinquagenarian insomnia.
\end{abstract}

\section{Introduction}

With the development of human beings' overall health, psychosomatic disorder which is stood by insomnia is becoming an important public health problem and attains a high attention from medical community all over the world. Modern medical points out that treatment of insomnia should focus on eliminating the pathogenesis and synthesizing the means of treatment,which contents mainly of psychological health education,CBT and medicine treatment especially the medicine is mainly the BZD. But these medicine easily causes the addiction and rebound insomnia, they get a limit of clinical use ${ }^{[1]}$.

WHO payed a lot of attention to the treatment of insomnia and raise inductive principals like treat on demand and small doses of intermittent treatment one after another..Issue about the dependency and patience is still a problem among both domestic and overseas medical community thus exploring a non-medicine way of treating insomnia is a good direction to update iatrotechnics.Some studies pointed out that EBS could prevent the side effect and addiction that medicine sleep disorder take brings them ${ }^{[2]}$.

\section{Objects and methods}

\subsection{Objects}

Choose the insomniacs who are received by our hospital and under our treatment during Jan,2016 to Oct,2016.After finishing the standard diagnosis Choose 80 by toss method patients and divide them into experimental group and matched group at random.Diagnose by the standards of insomnia in ICT-10 and exclude patients with severe somatopathy intemperant.Besides we have to attain permissions of patients or guardians before the study. The experiment grouping design is shown Table1. 
Table 1 experiment grouping design.

\begin{tabular}{|c|c|c|c|}
\hline group & male & Female & Average age \\
\hline Experimental group & 24 & 24 & $57.21 \pm 5.17$ \\
\hline Matched group & 16 & 16 & $56.90 \pm 5.12$ \\
\hline
\end{tabular}

Compare on general data like genders,ages,courses of disease and so on between two groups above has no statistics meanings.

\subsection{Research Methods}

\subsubsection{Methods}

Two groups of patients both take routine medicine--taking estazolam tablets $2 \mathrm{mg}$ a time.Take it orally half an hour before sleep and the course of treatment is about 4 weeks.Besides medicine the experimental group is with the EBS therapy(Nanjing hua wei medical equipment co. LTD, HW7002B Electroencephalography stimulation therapy).During the treatment patients should take a comfortable sitting position and clinostatism.Electrode slides will be attached to the patients'mastoid process of each ear nut.Electrode frequency will be set to $180-200 \mathrm{~Hz}$ and strength will be set to $80-100 \%$.Ratio is $1: 2$. Treatment will be taken once a day during the next 4 weeks and last for 30 minutes.

\subsubsection{Evaluation standard}

Evaluation tools:Adapt PSQI to evaluate the effect of treatment.The score is among 0 and 21,and the higher the score is the severer the problem of sleep is.Take evaluation respectively before and after the treatment, which can compare the two groups of patients' change of sleep quality.

PSG monitoring:Use PSG to monitor patients' sleep quality and monitor their sleep quality respectively two days after and before treatment is done.

\subsubsection{Statistical method}

Use SPSS16.0 to analyze the data we get and the measurement data will be tested by t.Regarding $\mathrm{P}<0.05$ as the difference has the statistic meaning.

\section{Results}

\subsection{PSQI scores}

The result of PSQI scores with before and after treatment is shown in Table2.

Table 2 Comparing two groups of patients'PSQI scores with before and after trea tment.( $\mathrm{x} \pm \mathrm{S}$ )

\begin{tabular}{|c|c|c|c|c|}
\hline events & \multicolumn{2}{|c|}{ experimental group } & \multicolumn{2}{c|}{ matched group } \\
\hline & before & after & before & after \\
\hline Sleep quality & $2.07 \pm 0.93$ & $0.92 \pm 0.78$ & $1.84 \pm 0.92$ & $1.43 \pm 0.19$ \\
\hline Sleep latency & $2.42 \pm 0.79$ & $1.48 \pm 0.70$ & $2.66 \pm 0.91$ & $1.46 \pm 0.95$ \\
\hline Sleep time & $1.73 \pm 0.86$ & $0.73 \pm 0.24$ & $1.65 \pm 1.11$ & 1.31 士 0.89 \\
\hline Sleep efficiency & $2.28 \pm 0.67$ & $0.90 \pm 0.74$ & $2.11 \pm 1.27$ & $1.49 \pm 0.75$ \\
\hline Sleep disorders & $1.88 \pm 0.82$ & $1.19 \pm 0.57$ & $2.22 \pm 0.94$ & $1.89 \pm 0.71$ \\
\hline hypnotic & $2.15 \pm 0.52$ & $0.49 \pm 0.33$ & $2.20 \pm 0.64$ & $1.74 \pm 0.88$ \\
\hline Day surgery & $2.41 \pm 0.72$ & $0.91 \pm 0.54$ & $2.24 \pm 0.88$ & $1.72 \pm 0.56$ \\
\hline Total & $16.68 \pm 1.65$ & $5.73 \pm 2.34$ & $14.80 \pm 2.17$ & $9.59 \pm 3.07$ \\
\hline
\end{tabular}

\subsection{Results of polysomnography(PSG)}

The results of comparing two groups of patients with sleep process is shown in Table3. 
Table3 Comparing two groups of patients with sleep process

\begin{tabular}{|c|c|c|c|c|}
\hline events & \multicolumn{2}{|c|}{ experimental group } & \multicolumn{2}{c|}{ matched group } \\
\hline & before & after & before & after \\
\hline Total sleep time & $320.13 \pm 87.20$ & $548.20 \pm 68.79$ & $320.97 \pm 91.40$ & $642.46 \pm 41.56$ \\
\hline Sleep latency & $53.23 \pm 21.22$ & $22.38 \pm 16.11$ & $54.04 \pm 23.14$ & $33.35 \pm 12.24$ \\
\hline Awakening time & $103.11 \pm 36.21$ & $41.32 \pm 22.06$ & $104.32 \pm 34.57$ & $61.35 \pm 28.67$ \\
\hline $\begin{array}{c}\text { Sleep } \\
\text { efficiency(\%) }\end{array}$ & $61.84 \pm 14.56$ & $91.57 \pm 13.89$ & $62.54 \pm 21.58$ & $81.35 \pm 21.69$ \\
\hline
\end{tabular}

Results showed that both two groups of patients' sleep progress have been improved a lot after treatment and has a noted difference $(\mathrm{P}<0.05)$ comparing with the one before treatment,which appears an excessive sleep,a shortening of sleep latency,a shortening of awaking time and an improve in integrity of sleep efficiency.Comparison between the two groups showed that the index in the treatment group in the process of sleep (total sleep time, sleep efficiency, sleep latency and awakening time) were superior to control group, comparing the two groups have significant difference $(\mathrm{P}<0.05)$.

\section{Discussion}

Insomnia is a kind of common disease, frequently-occurring disease and refractory disease, along with the accelerating pace of life, people all kinds of stress are also increasing, people suffering from insomnia is a trend of gradual growth.Plenty of sleep to maintain the balance of a person's mental and physical state is of great significance ${ }^{[3]}$.At present, based on people's cognition to the sleep patterns, use of magnetic stimulation of non-toxic, painless, security and development of the application of magnetic stimulation device therapy in the treatment of sleep disorders in has achieved good effect.EBS is a relatively safe and effective treatment technology.EBS is through imitation bioelectricity current digital frequency synthesis technology, and through the skin to stimulate the cerebellar nucleus, so that the capillaries in the brain blood flow, improve cerebral microcirculation, and at the same time around the brain produce certain frequency electromagnetic fields, and through the magnetic coupling coil way to patients, make the brain produces the synchronization effect, ultimately play a positive role in inducing sleep. The physiology of main explanation for the mechanism of sleep: sleep is made up of two alternating fast wave and slow wave sleep sleep phase process, concrete can be divided into: sleep period, shallow sleep period, medium stage and depth from four periods.The moderate sleep and deep sleep the biggest impact on the quality of sleep.The elderly because of sleep duration caused by brain function change, and make the deep sleep time, and have trouble falling asleep, wake up, the problem such as shorten sleep time, show the obvious sleep disorder and a series of emotional problems ${ }^{[4]}$.

The current treatment of sleep disorder is relatively single, basic on drug treatment is given priority to, but because of addiction, and other side effects, drug treatment made in the process of treatment in patients with psychosomatic under high damage.Patients because of doubts and resist drug attitude makes the effect of the medication is not ideal and more easy to occur repeatedly.Diversified therapy is currently an important trend of sleep disorder treatment, as the electromagnetic treatment technology is increasingly applied in all kinds of clinical disease, people have gradually accepted the application of this technology, eeg bionic electrical stimulation was based on this, the clinical application.Principle of the equipment is mainly through the alternating magnetic field to function in the brain's temporal lobe, occipital lobe and frontal lobe, and stimulation of cerebellar fastigial nucleus in the form of bioelectricity, and ultimately affect the brain neural electrophysiological activity, improve its mechanism is mainly for the ion permeability, promote the micro environment of brain cells, cause changes in the excitability of nerve cell, adjust the excitement and inhibition process of cerebral cortex, improve brain tissue on the regulation of autonomic nervous function, correcting for autonomic nerve dysfunction caused by insomnia and related physical symptoms, achieve the goal of treating insomnia restore brain function. 


\section{Conclusion}

In this study through the eeg bionic electrical stimulation with drug therapy can effectively eliminate the patient's insomnia, and no significant side effects, the method can be further used in clinical. Limited to the study area, and the characteristics of the sample, this study method also need to be perfected further, and get the relevant neural biology technology support.

\section{Acknowledgements}

Fund project: science and technology research project in Heilongjiang province department of education (Nomber: 2016-KYYWF-0882)

\section{References}

[1] Song Qiuyun.(2017)EBS with massage for the treatment of sleep disorder after stroke.Medical equipment,30(17),114-115.

[2] Yun-Yan H E, Zhong-Ping A N, Jin Y, et al.(2012) The influence of comprehensive nursing on ambulatory blood pressure in stroke patients with sleep disorder. Tianjin Journal of Nursing, 20(6),359-360.

[3] Ruberto M, Liotti F. (2011)Obstructive sleep apnoea syndrome (OSAS) and mood disorders in a population of public transport drivers. Medicina Del Lavoro, 102(2),201-207.

[4] Zhdanova I V. (2004)Advances in the management of insomnia. Expert Opinion on Pharmacotherapy, 5(7),1573-1579. 